\title{
Téoros
}

Revue de recherche en tourisme

\section{Le tourisme au pays du multimédia : pour s'y rendre, comment s'y prendre ? quoi y faire?}

\section{Louise Guay}

Volume 14, numéro 3, automne 1995

Tourisme et technologies de l'information

URI : https://id.erudit.org/iderudit/1075083ar

DOI : https://doi.org/10.7202/1075083ar

Aller au sommaire du numéro

Éditeur(s)

Université du Québec à Montréal

ISSN

0712-8657 (imprimé)

1923-2705 (numérique)

Découvrir la revue

Citer cet article

Guay, L. (1995). Le tourisme au pays du multimédia : pour s’y rendre, comment s’y prendre ? quoi y faire ? Téoros, 14(3), 22-25.

https://doi.org/10.7202/1075083ar d'utilisation que vous pouvez consulter en ligne. 


\title{
LE TOURISME au PAYS DU MULTIMÉDIA POUR S'Y RENDRE, COMMENT S'Y PRENDRE? QUOI Y FAIRE?
}

\author{
Louise Guay
}

\section{Louise Guay \\ est présidente de Public \\ Technologies Multimédia Inc. \\ (e-mail: louiseg@cam_org) \\ (http://wwwvirtual \\ marketplace.com/}

\section{Le Marché Virtuelmic} Marché Virtuel, marché
mondial et tourlsme

De nouveaux marchés s'ouvrent dans toutes les directlons et de nouveaux compétiteurs apparalssent. La recherche de clients et de partenaires stratéglques afln de mleux rencontrer les défls du manché global est plus active que Jamals. Et II n'y a pas de temps à perdre ! L'industrie du tour'lsme, dans les transformations quil l'affectent, est fortement concernée. Les entreprises sont Invitées à pénétrer au cceur d'un marché mondial, actif en tout lleu jour et nult.

Le Marché Virtuel est un outıl de marketing stratéglque qul représente les entreprlses à travers le monde sur Internet. CD-ROM et dans des klosques interact.fs, à l"alde de la pulssante technologle multimédla ${ }^{1}$. II allle les plus récentes technologles de communication et des techniques de marketing aggresslves et créatrices pour effectuer une prospectlon effcace du marché et alder les gens à falre des affaires partout dans le monde, rapidement et à un coût abordable.

\section{Un outll intégré}

Le Marché Virtuel est blen plus qu'une brochure corporatlve ou un représentant, et blen davantage qu'une présentation multimédla, une folre commerclale ou un accès Internet. C'est tout cela et plus encore ! Le Marché Virtuel est un outıl de marketing bl-directionnel qul permet aux entreprises de se présenter, de même que leurs prodults et Intérêts, à l'alde de la pulssance multimédla. Il multiplle la portée de leur force de vente, accroit la visibilité de leurs actlvités et permet aux clients et partenalres de communlquer entre eux sans délal.

De plus, Il offre un feed-back permanent par l'Intermédlalre de statistlques d'uttlisation. Pour le voyageur, c'est l'accès direct aux ressources touristlques. Pour l'agence de voyage, c'est l'ajout à ses services du rôle de gulde-anlmateur : une dimension riche et profonde qul l'implique au cceur du processus tourlstique. Pour I'y alder, un logiclel Interactif sera utilisé afin de mleux clbler les besolns des voyagenrs.

\section{Une présentation pulssante}

Le Marché Virtuel est composé d'une mulutude de présentatlons dynamlques qul s'intègrent dans divers secteurs. Chaque présentation est composée d"une animation de haute qualité, d'une sonorisation, de graphlques et de textes pour décrire au mleux l'entreprlse, ses prodults et ses intérêts stratégrques. Les cllents sont en mesure d'effectuer des recherches par secteurs d'Industrle, noms de compagnle prodults et mots-clés. Dans un avenir rapproché sera cependant offerte une fonction de recherche intelligente qul permettra une connalssance très flne des cllents.

Lorsquils ont trouvé une Information d'intérêt, les cllents peuvent regaruler une présentation, Imprimer une brochure et Ident.lfler la personne-ressource pour une communlcation dlrecte. Cette interaction est hautement propice au marché du tourlsme et permet au voyageur de clbler efflcacement ses besolns et de les partager avec son agent de voyage.

\section{En lout temps et tout Ileu}

Les utillsateurs peuvent alnsi trouver de nouveaux prodults, de nouveaux vendeurs, distributeurs et partenalres. L'utlité ne pourrait ểre plus grande. Le Marché Virtuel est distribué aux publıcs-cibles à l"alde d'un CD-ROM et sur la partle graphlque du réseau Interuet. appelé World Wide Web. Chaque entreprise bénéflcle de l'important volume d'actuvité sengendré par le regroupement d'une industrle entlère en un même lleu. Le résultat est une folre commerclale virtuelle accessible par ordinateur en tout temps. C'est pour le tourisme une fenêtre sur tous les pays du monde.

\section{Une mise en marché aggressive et ciblée}

Les techulques de marketing traditionnelles et numérlques assurent que le Marché Virtuel solt vu par les cllentèlesclbles. Des campagnes de promotion imprimée et électronlque dans la presse commerclale, du marketing direct, de la publlclté, des folres commerclales, des organisations commerclales gouvernementales et. une distribution Internet sont mIs en ceuvre pour positionner efficacement les entreprises sur le marché.

Le klosque Interactif du Marché Virtuel est présenté dans les folres commerclales majeures partout dans le monde afin d'offr'r aux entreprlses une présence qui rapporte blen davantage qu'un déplacement réel et con̂teux. Incluant un écran tactile, un téléphone et un télécopleur, Il permet des contacts personnels Immédiats. La vidéo-conférence est également offerte en optıon.

\section{Un outil complémentalre quf rapporte}

Le Marché Virtuel complète bien les activités de marketung régullères d'une entreprise : brochures, folres commerclales ou site Internet, tout en ne coûtant quime fraction d'une seule d'entre elles. Le retour sur l'Investissement est rapide : une question de semaines. Abordable et efflcace, le Marché Virtuel prospecte en tout temps et continue à rapporter blen au-delà de la mise initlale. C'est une opportunité à salsir et quil permet d'accroitre la richesse de contenu de tout secteur Industrilel, y comprls blen sûr celul du tourlsme. 


\section{2- Contexte}

\section{1- Points de vue du tourisme}

\section{Tourlsme et technologle de l'information}

A l'heure de l'inforoute, I'industrle touristlque s'organ Ise, s'équipe et se branche au multimédla. Actuellement, ces deux industries sont celles dont la crolssance est la plus raplde et la plus dynamique. Encore et toujours pourrait-on dlre, car les gens n'ont eu de cesse de voyager depuls que nos loIntains ancêtres ont appris à marcher, et lls ont touJours Innové afın d'améllorer leurs conditlons de vle. Question contexte, soullenons que cette industrle représente 3.4 trillions de dollars US.

\section{Révolution et défis}

Le monde du tourisme se trouve à sublr une transformation radicale. En effet, l'uttlisatIon des technologles de I'Information pour mleux organlser, promouvolr et commerclallser les prodults et services touristlques. entraîne d'importantes répercussions qul Iront jusqu'à toucher les fondements même de cette Industrle. II s'agit en falt d'une révolution technologique dont les effets seront plus importants encore que l'apparition des avlons à réaction.

Partout dans le monde et à tous les nlveaux. les Intervenants llés au tourlsme ouvrent de pair avec les producteurs d'outlls et services technologiques. afin d'apporter des solutions concrètes et efflcaces aux problèmes actuellement posés par le tourtsme. II Importe de répondre adéquatement aux nouveaux défls du marché, en offrant une structure nouvelle. basée sur l'organlsation électronique du marché du tourisme. Cette transformation se fera par étapes successives et mènera l'Industrle vers des sommets inégalés.

\section{Un marché électronique}

La synergle des technologles de l'Information et des télécommunications augmente de façon considérable la capacité de traltement de I'Information, de même que la quantuté et la qualité de solutions que l'on peut proposer aux divers intervenants. En fait, on peut afflrmer sans exagératlon que l'accès au marché tourlstıque sera dorénavant de nature élec- tronlque et que ceux qui ne foueront pas cette carte risquent fort de ne plus être de la partle. C'est toute l'Industrle qul se met à l'ère des technologles de l'Information.

II Importe aussi d'examlner plus en détall deux obstacles majeurs à la commerclallsation de toute la gamme des prodults tourlstlques sur le marché électronlque, solt la concurrence entre les gouvernements et les entreprises privées et les désaccords sur les procédures à sulvre pour le fonctionnement d'un réseau mondlal consacré au tourisme. II faudralt donc favorlser le partenarlat stratéglque entre les gouvernements et l'industrle, et imaginer des solutions pratiques et valables pour tous quant à la mise en marché électronlque du tourisme.

\section{2- Points de vue du} touriste

Une approche persomnallsée

Vollà qu"en plus les tourlstes eux-mêmes se dotent maintenant. d'outlls multimédla perfectlonnés, performants, qui s'adaptent aux gouts de chacun, pour les alder à mleux voyager. La technologle envahit peu à peu le cceur et la réallté quotidlenne des gens. On offre maintenant aux tourlstes des kits de voyage pour les alder à se rendre là où 118 veulent aller... et pour être capable d'en revenir ! Mals avant le départ. II y a aussı la planiflcation : le plalsir vient non seulement du voyage, mals de savolr de quol Il sera falt. Dès lors, l'Important est de sortir des sentlers battus, de créer son voyage personnallsé, d'y aller à sa façon. Bref. le tourisme, c'est l'aventure. Et pour mleux vous y engager, volcl des outils plus sophistiqués les uns que les autres.

\section{Des outils technologiques}

Le fameux système GPS (global poslttonIng system) - ou système de postlonnement global - est une percée technologique récente offerte aux grands voyageurs et navlgateurs. Il s'aglt diun ordlnateur portatif quil permet à une personne de détermlner sa positton géographlque exacte, où qu'elle solt de par le monde. Le GPS utllise les ressources d'une base de domées cartographlque élaborée et. se rellant aux satellites en orblte, Iocallse Immanquablement l'aven- 
turler. On peut également demander au GPS de tracer tout le chemin déjă parcouru, question de sécurité ou d'encouragement.

Il exIste aussI des CD-ROMs qul se veulent à la fols un atlas, un navigateur, un coordinateur d'itinéralre et un planiflcateur d'actlvités. Bref, un "falseur" de voyages. Vous Indlquez vos projets et le logiclel vous concocte une destination et un Itınéraire sur mesure, Incluant la mellleure route à sulvre pour bénéflcler des mellleures activités offertes. Voyage hautement personnalisé garantı, partout au Mexıque, au Canada et aux Etats-Unls. Il ne vous reste plus qu’à falre les bagages !

Compacte, légère et réslstante, la montre de voyage s'adresse quant à elle à ceux quI n'alment pas encombrer leurs déplacements. Cette montre falt alnsl office de compas, d'altimètre, de baromètre et de thermomètre. Falt remarquable, elle donne également l'heure ! Les aventurlers des mers seront heureux de pouvolr l'utlliser de palr avec un compas numérique, fonctionnel même au sein du redoutable trlangle des Bermudes. Entre autres avantages. II corrlge les distorsions magnétlques provenant du bateau et enreglstre tous vos déplacements, vous permettant alnsl de toujours rentrer à bon port.

La technologle s'harmonise donc au mleux, et parfols même de façon fort Intultive, avec les besolns des voyageurs. II s'aglt d'une ouverture au monde, une interaction personnaIIsée et créatrice. Les technologles de l'Information préludent donc à une Industrle du tourisme renouvelée, en utllIsant tous les chemins quil lul sont offerts.

\section{3- Lieux et chemins}

\section{1- Linitiation au voyage Une vision nouvelle}

Il y a plusleurs façons de voyager et même de salsır la nature réelle du voyage : chacun personnallse ses expérlences selon son vécu. Mals la technologle et plus partilcullèrement le multimédla interactif propose une vislon nouvelle et partagée par tous du tourlsme. Le voyage n'est plus limité dans le temps et dans l'espace. Ses frontlères s'ouvrent vers de nouveaux horizons, de nouvelles appllcatlons.

\section{La cartographle multimédla}

C'est toute la notion de cartographle qul est. révisée et améllorée. La carte est l'interface de communication multimédla la plus naturelle quI soit, Impllquant une relation et Influence réclproque entre le voyageur et son parcours, entre le tourisme et le monde. La carte-pllote est l'interface par' excellence pour les appllcatlons multimédla. C'est le touriste-voyageur qui donne alnsl le ton.

\section{Chemins dans I'espace et le temps}

Le tourisme el le multimédla Invitent tous deux à des déplacements, l'un dans l'espace plysique et lautre dans l'espace virtuel. Il s'agit pourtant dans les deux cas du corps humain et de ses destinations. Les chemlns du touriste à travers le temps et l'espace ont des recoupements étonuants. Les routes sulvent un chemin à travers les âges qui demeure en grande partle le même. C'est la route à suivre en directlon d'une éminence reconnalssable. Les mellleurs guldes de voyage sont alnsi des leçons de choses, des exerclces d'observatlon, des enquêtes du réel qui, à partir d'indices insigniflants, permettent de reconstrulre le monde, tel un vlllage, une place, une fontalne, une malson, ou même des sérles d'évênements.

\section{Le voyage par les médlas}

Les nouveaux médlas font voyager et transportent par leur capacité à alder le voyageur à falre son propre chemin dans la connalssance qu'lls lul proposent. Donc de découvrir l'environnement par ses propres moyens, d'apprivolser le génle du lleu, d'effectuer des voyages personnallsés, adaptés à l'utIllsateur de la technologle, En d'autres mols, Ils permettent d'effectuer des voyages intérieurs à la surface des it:inéralres extérleurs solgneusement préparés.

Le voyageur acqulert également le contrôle. C'est lul qul dorénavant pllote sa destination, décide de planifler ses actlvités et d'orchestrer ses gôtts avec ce qui s'offre à lul. La technologle lul confère la responsabilité du cholx. Il se prend en charge et se volt offrir la carte interactive des lleux, des réglons, véritable parcours et sıte d'exploratıon en ellemême. Le multımédla et ses interfaces sont dorénavant comparés à un océan dans lequel on navigue, sur lequel on

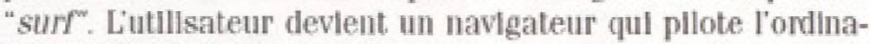
teur et même dans certains cas une sorte de valsseau spatial.

Les lleux virtuels sont polymorphlques, mouvants, tremblants, vibrants. Comme le sont les lleux physiques lorsque le voyageur est Inspiré. Le vral voyage est toujours celul du voyageur solitalre quil réinvente à chaque fols le lleu parcouru, malntenant alnsı à travers les époques les llenx et les chemins quI les relient. Ce voyageur est dit solltalre, étant. celul quil falt son chemln à sa façon, dans le but d'établlr des relations de mellleure qualité avec tous les autres voyageurs. D'allleurs les plus beaux voyages sont ceux que des amls nous ont falt découvrir et quil rejolgnent nos gonts les plus personnels, volre secrets.

Un voyage comporte toujours une étude et des outlls de recherche, des apparells et Instruments quil le rendent passlonnant, vlvant et rellé à l'hlstolre passée, présente et future des llenx. Le voyage redevlent une expérlence, une inltlation personnelle, un dépaysement, un apprivolsement de l'Inconnu.

\section{2- Le tourisme virtuel \\ De nouveaur rôles}

Le inultimédla et les réseaux électronlques jettent les bases pour reller tourisme et tourlste. Les rapports entre eux risquent alnsı d'être Inversés : la balance du pouvolr favorlsera désormals le touriste, qui devlent un voyageur, explorateur, aventurter, bien Informé et préparé. Par son accès au réseau unlversel qu'est. l'inforoute, II rencontre dlrectement les gens et. ressources qul l'Intéressent. II est l'Instlgateur du processus. Liagence de voyage agit dès lors à plusleurs titres : guide-accompagnateur. fureteur d'Informations sur les réseaux, etc., facilitant la rencontre du voyageur et de sa destination. laquelle va d'allleurs en se rafmuant. Citoyen unlversel. le voyageur alme conquérll la nature par des 
activités peu communes et audacleuses qui ne compromettent pas l'environnement. Ses parcours dans les villes sont ceux d'une personne dont la culture est basee sur la rencontre, l'échange et les contacts humalns : toute chose que le multimédla et les espaces virtuels permettent.

Un CD-ROM multimédla récemment mls sur le marché et développé pour les agences de voyage canadlennes falt un premler pas en effectuant le transfert et l'intégration de servlces déa offerts sur un support de haute technologle. Le Marché Vrtuel en effectue un second et repousse les limites du tourisme en proposant une nouvelle façon de falre les choses, une mise en contact directe. II falt donc blen plus que réorganiser l'information. Il la redéfinit en fonction des besolns du marché. Liagence de voyage est alnsl appelée à ouvrer encore davantage au nlveau du contenu personnallsé et à approfondir la relation avec le cllent.

\section{Préparatlon et rencontres}

Le Marché Vrtuel, par son Interaction continue, permet de débuter le voyage. La communication sur le réseau rend alnsi possible des échanges d'informations pertinentes sur les lleux d'Intérêt et même des témolgnages dlrects. Le tourlsme possede a cet égard une profonde dimension de préparatlon, qu'elle solt d'ordre matérlel, culturel, spirituel ou autre.

Un déplacement virtuel permet alnsi de se mettre en relation et cette préparation permet une mellleure compréhension du lieu où I'on va. Donc une appréclation plus riche. plus contrastée, et un comportement plus adéquat en regard des contungences et des valeurs rencontrées par le voyageur. Par exemple, Il seralt possible à cet effet de rencontrer sur le réseau des gens de divers pays de par le monde qul voudralent blen accuellilir les visiteurs, les renselgner, discuter avec eux de leurs désirs et vislons.

En bref, Il Importe de transcender la simple notion de tourlsme pour approfondlr le concept de voyage. Le voyage est une rencontre à laquelle il Importe de se préparer. C'est la transformation du touriste en un véritable artiste créateur de son voyage. Liarchitecture du Marché Vrtuel et la pulssance du réseau non seulement permettent mals favorisent le contact entre dlvers lleux et différentes cultures. C'est une Invitation personnalisée vers d'autres horlzons et à déflnir son propre parcours.
Note:

1 Le multimédia est une industrie de contenu quil intègre tous les medla et leurs supports. II est. l'́cosysteme naturel de lordinateur et de ses réseaux. se voulant à la fols un langage, un processus et un systeme. Il peut être comparé,

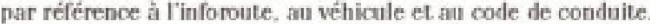

